\title{
A Numerical Evaluation Method of Non-uniformity in Hollow Torsional Test
}

\author{
Binbin $\mathrm{Xu}^{1, \mathrm{a}}$ and $\mathrm{Wei}, \mathrm{Si}^{2, \mathrm{~b}}$ \\ ${ }^{1}$ Tianjin Port Engineering Institute Ltd. of CCCC, Tianjin, China; Key Lab. of Geotechnical \\ Engineering of Tianjin, Tianjin, China; Key Lab. of Geotechnical Engineering, Ministry of \\ Communication, Tianjin, China \\ 2 Tianjin Port Engineering Institute Ltd. of CCCC, Tianjin, China \\ axubinbin@tpei.com.cn, bsiwei@tpei.com.cn
}

\begin{abstract}
Keywords: Non-uniformity, Hollow torsional test, Numerical analysis, Evaluation index
Abstract. In order to get a perfect element-wise response to verify the basic mechanical properties of one soil, a hollow torsional test is usually preferred because its stress condition is more similar to the element response. However, during the torsion process, there is still more or less non-uniformity influencing the mechanical response. In order to evaluate the non-uniformity of each variable quantitatively, a new method is proposed taking the deformation of the whole elements inside the specimen and the results show that it is suitable to evaluate the non-uniformity in the hollow torsion test.
\end{abstract}

\section{Introduction}

In order to clarify the soil properties and verify the validity of proposed constitutive model, various in-door experiments have been carried out to check the mechanical behavior of soil under different stress path, such as plane strain test, oedometer test and triaxial test, in which the triaxial compression test is the most popular test. However, the stress state in the triaxial test is not three dimensional and it cannot be called a true triaxial test. In practical geotechnical engineering, the soil in the ground is usually under a three dimensional stress state. Recently, the hollow cylinder test that is determined by four individual stresses is employed to simulate the practical stress state during the construction.

Specimen in laboratory testing including the above hollow cylindrical specimen is usually regarded as a single element possessing uniform deformation to verify the validity of constitutive model. However, as the progressing of loading, such uniformity will lose gradually, which may leads to inhomogeneous and localized deformation. Asaoka and Noda [1] described the specimen as a boundary value problem with initial conditions under plain strain assumption and pointed out the importance of considering the effect of boundary value problem specially in the case of rapid loading and large deformation. Jin et al. [2] dealt with a three dimension triaxial test regarded as a boundary value problem and demonstrated the difference between soil specimen and the perfect element concerning cyclic frequency, confining pressure and so on using quasi-static FE analysis code.

However, during the hollow torsional test the deformation is not always uniform and there would be more or less non-uniformity. In the practical indoor experiments, because the specimen in installed inside the cell and it is impossible to measure the variation of uniformity during the torsional process. However, in the numerical experiments, we can see the non-uniform deformation obviously [3], and the question is how to evaluate the extent of non-uniformity.

\section{Calculation of Variables in Hollow Torsional Test}

In practical process, the entire specimen is regarded as a single element with stress and strain components derived from an assumption of linear elastic constitutive equation and infinitesimal deformation. Eqs. 1, 2 and 3 present the average stress and strain, where $R_{o}$ and $R_{i}$ are the external and internal radius; $u_{i}$ and $u_{o}$ are the inner and outer displacement; $\Delta H$ and $\Delta \theta$ are the increments in vertical and circumferential direction. For the shear stress $\sigma_{\theta z}$, there are two assumed distributions: 
one is elastic distribution $\sigma_{E}$ with linear increase and another is completely plastic distribution $\sigma_{P}$ with a uniform value, which results in the two different calculation methods as shown in Eq. 1. Here, $\delta$ is the percentage of plastic distribution and always taken as 1 unless state otherwise.

$$
\begin{aligned}
& \sigma_{z z}=\frac{F_{z}+\pi R_{o}^{2} P_{o}-\pi R_{i}^{2} P_{i}}{A} \\
& \sigma_{r r}=\frac{P_{o} R_{o}+P_{i} R_{i}}{R_{o}+R_{i}} \\
& \sigma_{\theta \theta}=\frac{P_{o} R_{o}-P_{i} R_{i}}{R_{o}-R_{i}} \\
& \sigma_{E}=\frac{T_{\theta}}{\pi\left(R_{o}^{2}+R_{i}^{2}\right)\left(R_{o}-R_{i}\right)} \\
& \sigma_{P}=\frac{3 T_{\theta}}{2 \pi\left(R_{o}^{3}-R_{i}^{3}\right)} \\
& \sigma_{\theta z}=(1-\delta) \sigma_{E}+\delta \sigma_{P} . \\
& \varepsilon_{z z}=\frac{\Delta H}{H} \\
& \varepsilon_{r r}=-\frac{u_{o}-u_{i}}{R_{o}-R_{i}} \\
& \varepsilon_{\theta \theta}=-\frac{u_{o}+u_{i}}{R_{o}+R_{i}} \\
& \varepsilon_{\theta z}=\frac{\Delta \theta\left(R_{o}^{3}-R_{i}^{3}\right)}{3 H\left(R_{o}^{2}-R_{i}^{2}\right)} . \\
& p=-\frac{\sigma_{z z}+\sigma_{r r}+\sigma_{\theta \theta}}{3} \\
& p^{\prime}=p-u_{e} \\
& q=\sqrt{\frac{3}{2}\left\{\left(\sigma_{z z}-p\right)^{2}+\left(\sigma_{r r}-p\right)^{2}+\left(\sigma_{\theta \theta}-p\right)^{2}+2 \sigma_{\theta z}^{2}\right\}} .
\end{aligned}
$$

\section{Evaluation of Non-uniformity in Hollow Torsional Test}

Hight et al. [4] defined a coefficient $\beta_{3}$ for each individual stress to evaluate the non-uniformity along the radial direction:

$$
\beta_{3}=\frac{1}{\left(R_{o}-R_{i}\right)} \frac{1}{\sigma_{L}} \int_{R_{i}}^{R_{o}}|\sigma(r)-\bar{\sigma}| d r .
$$

where $\sigma(r)$ is the real stress such as $\sigma_{r r}, \sigma_{\theta \theta}$ and $\sigma_{\theta z}, \bar{\sigma}$ is the average value of the corresponding stress and $\sigma_{L}$ is the standard stress taken as $\bar{\sigma}_{\theta z}$ for $\sigma_{\theta z}$ and $1 / 2\left(\bar{\sigma}_{\theta \theta}+\bar{\sigma}_{r r}\right)$ for $\sigma_{r r}$ and $\sigma_{\theta \theta}$. It is acceptable for $\beta_{3}<0.11$. Sayao and Vaid [5] proposed another evaluating coefficient $\beta_{\eta}$ viewed from the stress ratio:

$$
\beta_{\eta}=\frac{\eta_{\max }-\eta_{\min }}{\bar{\eta}} .
$$

where $\eta_{\max }, \eta_{\min }$ and $\bar{\eta}$ are maximum, minimum and average stress ratios respectively. It is acceptable for $\beta_{\eta}<0.20$.

However, Eq. 4 is proposed based on the axial-symmetry deformation only considering the difference between inner and outer pressures, which is not suitable for three-dimension analysis. 
Moreover, for both Eqs. 4 and 5, it can only evaluate the non-uniformity at designated combinations of stress state in which constant $\sigma_{L}$ and $\bar{\eta}$ are required to quantify the magnitude of $\beta_{3}$ and $\beta_{\eta}$, which restricts more general stress paths. Therefore, a new method with variable $\Delta A$ is defined as follows to assess the non-uniformity:

$$
\Delta A=\frac{\sum_{i=1}^{N E}|A(i)-\bar{A}| \cdot \Delta V(i)}{\sum_{i=1}^{N E} \Delta V(i)} .
$$

where

$$
\bar{A}=\frac{\sum_{i=1}^{N E} A(i) \cdot \Delta V(i)}{\sum_{i=1}^{N E} \Delta V(i)} .
$$

Here, $A(i)$ can be the mean effective stress, deviator stress, shear strain and so on within each element, $\Delta V(i)$ is the current volume for element $i, \bar{A}$ is the weighted average of $A(i)$ taking the volume ratio $\Delta V(i) / \sum_{i=1}^{N E} \Delta V(i)$ as the weight coefficient where " $N E$ " represents the number of elements, and $\Delta A$ is the weighted average of deviation between $A(i)$ and $\bar{A} . \Delta A$ has the same unit with $A(i)$. The smaller $\Delta A$ is, the more uniform $A(i)$ is. The ideal uniform distribution of $A(i)$, that is $A(i)=$ const, results in $\Delta A=0$.

\section{Example of Evaluating Method}

As can be understood above, the proposed method can take the non-uniformity of all the elements into consideration and it is a more precise method compared with the previous one. In order to verify the validation of this method, an example is given to show the variation of non-uniformity of each variable during the torsion process.

Fig. 1 illustrates the non-uniformity of mean effective stress $p^{\prime}$ and deviator stress $q$, stress ratio $\eta$ and shear strain $\varepsilon_{s}$ respectively as the apparent shear strain $\gamma_{s}$ increases, where the mean effective stress $p^{\prime}$ and deviator stress $q$ are normalized by the initial confining pressure. Two evaluation criterions have been put forward by Hight et al. [4] and Sayao and Vaid [5] based on the axial-symmetrical assumption. Here, the magnitude of non-uniformity is quantified by the deviation from the average values viewing from the entire specimen which is suitable for $3 \mathrm{D}$ deformation. It is easy to understand that the larger the deviation is, the severer the non-uniformity is. From Fig. 1(a) it can be seen that the deviation of $p^{\prime}$ is less than 0.11 which is acceptable according to $\beta_{3}<0.11$ defined by Hight et al. [4]. Meanwhile, the maximum deviation of $\eta$ in Fig. 1(c) occurs around $\gamma_{s}=4 \%$, which is also acceptable according to $\beta_{\eta}<0.20$ defined by Sayao and Vaid [5]. Therefore, the new evaluation method is able to take both of the evaluation indexes into consideration simultaneously under more general stress paths. If we look into the variation of deviation in $p^{\prime}$ specifically, the deviation firstly increases to a peak and decreases until near zero following by another increasing and decreasing. As can been seem, the variation of different variables are quite different from each other and the proposed method is proved to be very effective in the evaluation of non-uniformity. 


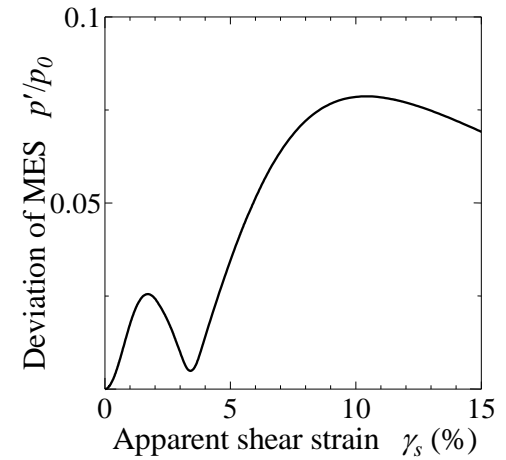

(a) Deviation of $p^{\prime}$

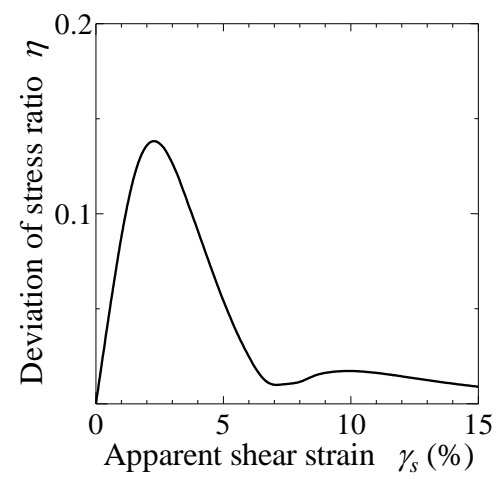

(c) Deviation of $\eta$

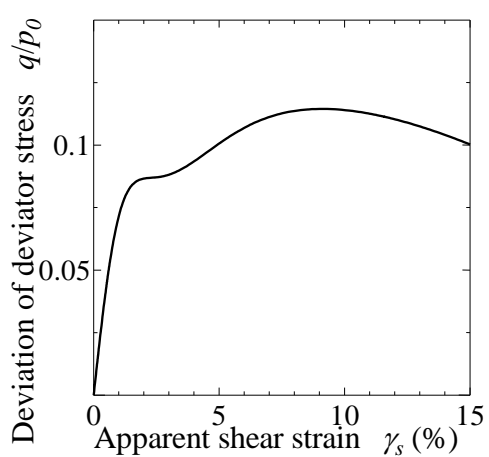

(b) Deviation of $q$

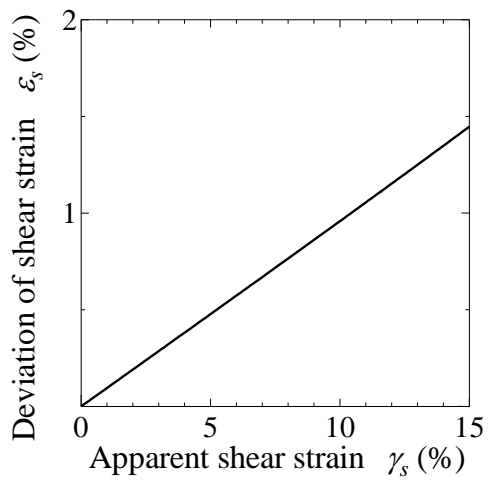

(d) Deviation of $\varepsilon$

Figure 1 Deviation of mean effective stress $p^{\prime}$ and deviator stress $q$, stress ratio $\eta$ and shear strain $\varepsilon_{s}$

\section{Conclusions}

As it is well known, the response of the hollow torsional test is the most close to the response of single element. However, there is still a little shortcoming about the experiment, that is, the non-uniformity often occurs during the torsion process and it influences the results significantly. In order to evaluate the non-uniformity of each variable quantitatively, a new method is proposed based on considering the deformation of the whole elements and the conclusions are as follows:

1) Different from the previous method where it can only consider the difference between inner and outer pressures and evaluate the non-uniformity at designated combinations of stress state, the proposed method can deal with the non-uniformity properly.

2) The variation of non-uniformity along with the shear strain can be seen clearly and it is easy to know the non-uniformity of variables at any torsional process.

3) The variation of non-uniformity for each variable is quite different from each other and it is reasonable to distinguish the different variables when carrying out the indoor experiments.

\section{References}

[1] A. Asaoka and T. Noda: Soils and Foundations, Vol. 35-1, (1995), p.83.

[2] Y. Jin, B. Ye and F. Zhang: Soils and Foundations, Vol. 50-1, (2010), p.177.

[3] B.B. Xu et al.: Advanced Material Research, Vol. 1065-1069, (2015), p. 317.

[4] D. W. Hight, A. Gen and M. J. Symes: Géotechnique, Vol. 33-4, (1983), p. 355.

[5] A. Sayao and Y. P. Vaid: Canadian Geotechnical Journal, Vol. 33, (1996), p. 822. 